Revue des patrimoines

\title{
Regard sur les méthodes d'analyse et de sauvegarde du patrimoine et de l'imagerie scientifique
}

Valérie Guesnier

\section{(2) OpenEdition \\ Journals}

Édition électronique

URL : http://journals.openedition.org/insitu/4054

DOI : 10.4000/insitu.4054

ISSN : 1630-7305

Éditeur

Ministère de la culture

Référence électronique

Valérie Guesnier, «Regard sur les méthodes d'analyse et de sauvegarde du patrimoine et de l'imagerie scientifique », In Situ [En ligne], 10 | 2009, mis en ligne le 19 mai 2009, consulté le 22 avril 2019. URL:

http://journals.openedition.org/insitu/4054; DOI : 10.4000/insitu.4054

Ce document a été généré automatiquement le 22 avril 2019

\section{(c) (i) () $\Theta$}

In Situ Revues des patrimoines est mis à disposition selon les termes de la licence Creative Commons Attribution - Pas d'Utilisation Commerciale - Pas de Modification 4.0 International. 
Regard sur les méthodes d'analyse et de sauvegarde du patrimoine et de l'imagerie scientifique

Valérie Guesnier

(fig. $\left.n^{\circ} 1,2,3\right)$ 
Figure 1

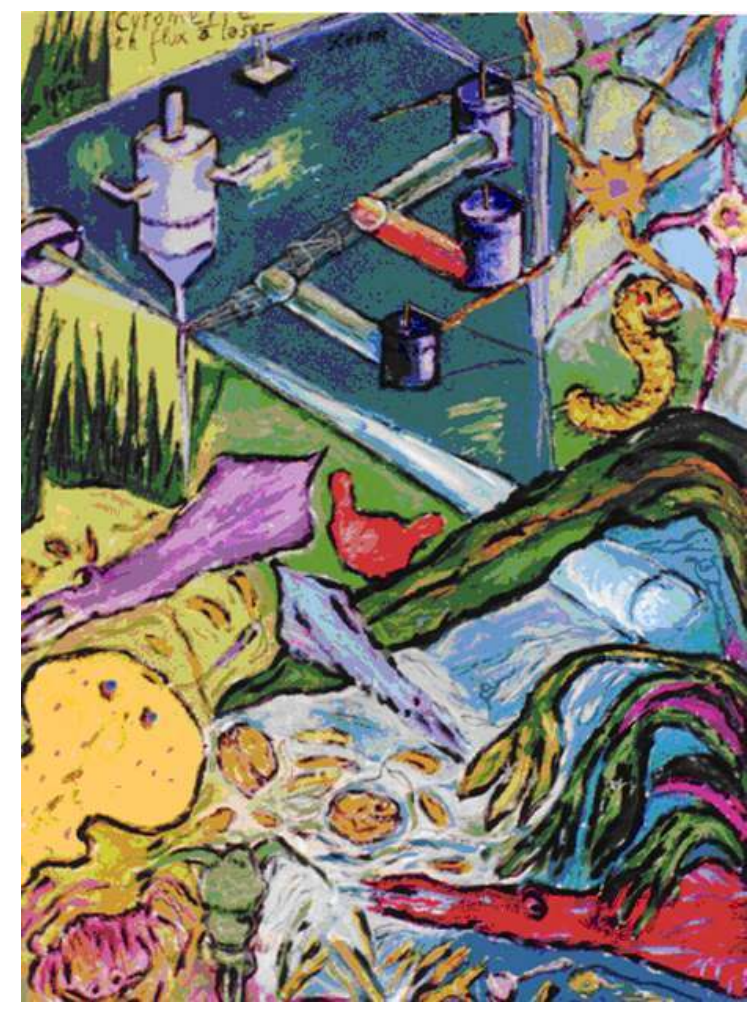

BIOLOgIE MARINE, panneau peint, Monique Peytral, 1989. Représentation par l'artiste d'un cytomètre en flux.

(c) Monique Peytral. 
Figure 2

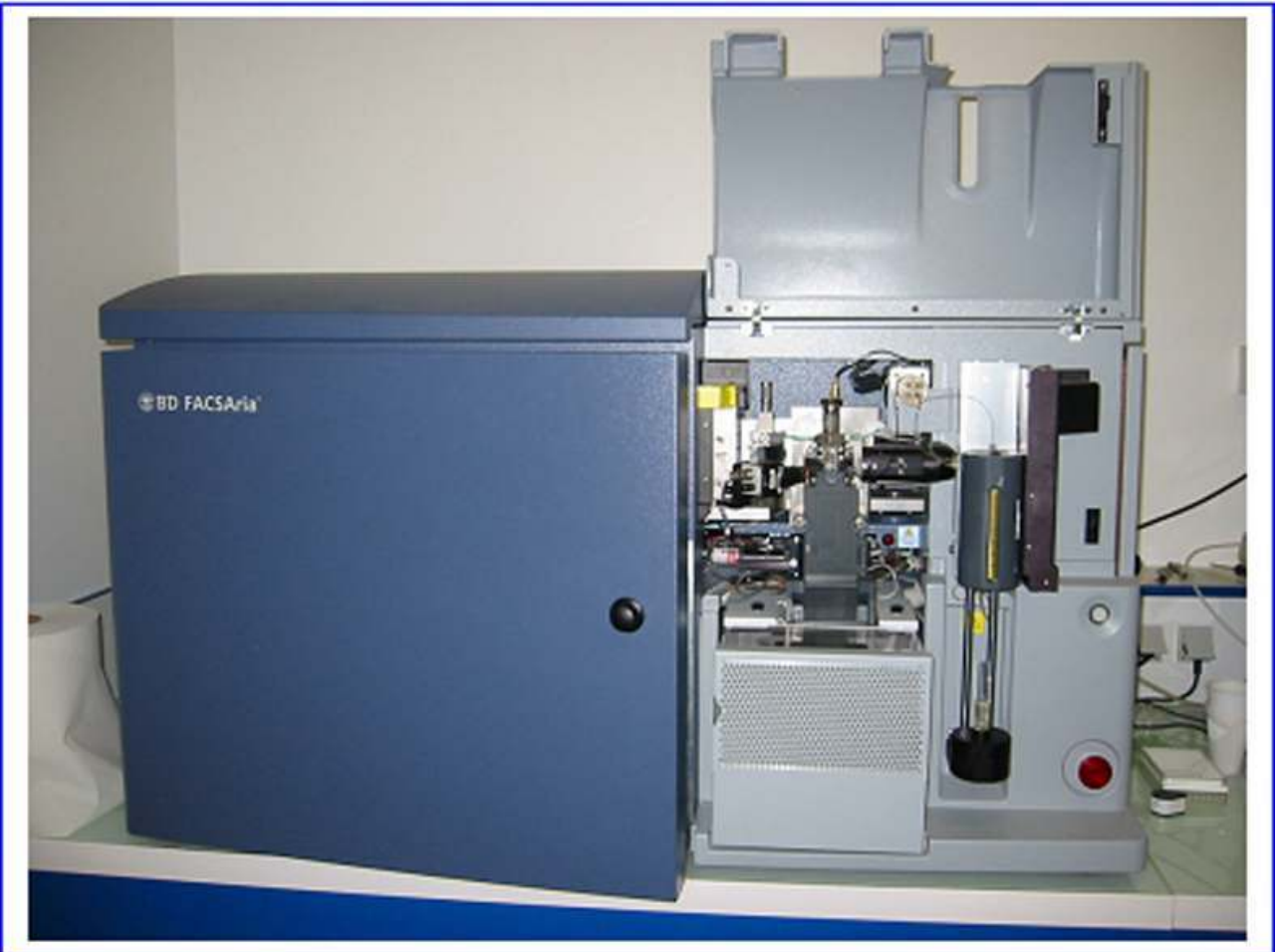

Cytomètre en flux. Instrument scientifique. Station Biologique de Roscoff. Phot. Valérie Guesnier. (c) Valérie Guesnier. 


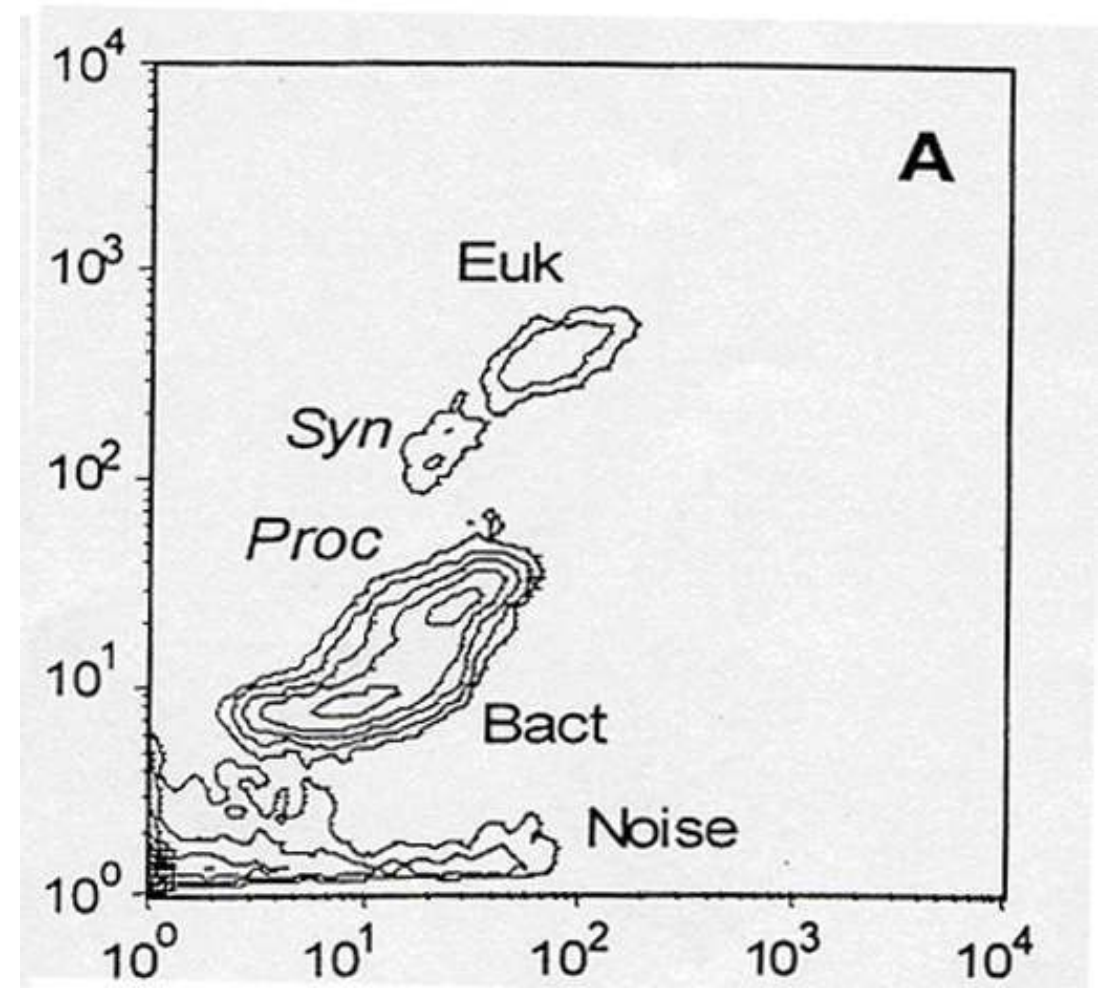

Image de cytométrie en flux. Graphique à 3D représenté en 2D. Moyens d'acquisition et d'analyse : cytomètre. Moyens de restitution : logiciel graphique de construction de l'image. In RAPPORT DE LA STATION BIOLOgIQUE DE ROSCOFF 1985-1990.

2 Étudier un établissement de recherche et d'enseignement en biologie marine et en

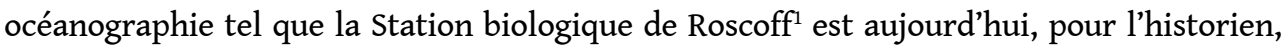
un exercice d'une relative complexité. Complexité tenant, avant tout, à un déficit d'outils méthodologiques que l'on peut imputer à une approche relativement lacunaire du concept de patrimoine scientifique.

Il est en effet apparu, au cours des recherches menées sur le patrimoine historique de la Station Biologique de Roscoff, que trois types d'éléments, liés par une relation de complémentarité sémiologique, constituent un héritage unique et indissociable: les éléments relevant du patrimoine immobilier tels que les bâtiments et, plus généralement, les diverses constructions nécessaires à l'exercice de l'activité scientifique, ceux composant le patrimoine mobilier, avec les équipements techniques et les instruments scientifiques, mais également les collections d'objets, les œuvres d'art, ou les documents iconographiques et l'imagerie scientifique ${ }^{2}$.

Ces trois types d'éléments patrimoniaux sont les traces matérielles des divers moyens mis en œuvre pour faire de la science, l'illustration du contexte historique qui la porte et qu'elle génère en retour, l'expression des résultats qu'elle produit. Mémoire tangible de l'histoire, ils permettent d'appréhender la science dans la diversité des paramètres dont elle est tributaire, qu'elle implique et qu'elle conjugue: humains, techniques, technologiques, logistiques, culturels, etc.

5 C'est la raison pour laquelle amputer le champ patrimonial d'un établissement scientifique d'une partie de ses éléments aurait pour conséquence de priver l'histoire 
d'une partie de ses faits. Pourrait-on, ainsi, restreindre l'étude d'un établissement à ses seuls bâtiments sans risquer de le réduire à une coquille vide privée de sa substance et de sa raison d'être: la science? Serait-il pertinent d'inventorier l'instrumentation scientifique sans l'associer à l'imagerie, cette restitution visuelle de la science, qu'elle permet d'obtenir? Quant au patrimoine artistique tel que portraits, monuments commémoratifs, décors monumentaux et autres compositions figuratives, décoratives, abstraites ou naturalistes, n'est-il pas le témoin matériel privilégié des personnalités, des événements, des découvertes, qui ont nourri l'histoire d'un établissement scientifique et en ont fait sa singularité ?

6 Cependant, malgré l'indéfectible lien de complémentarité unissant patrimoine immobilier, patrimoine mobilier et imagerie scientifique dans la compréhension et la restitution de l'histoire de la science et, par conséquent, dans la construction du concept de patrimoine scientifique, il apparaît aujourd'hui que l'historien n'est pas en mesure d'étudier l'ensemble des éléments constitutifs de ce domaine patrimonial. L'exemple de l'imagerie scientifique et, à un degré moindre, de l'instrumentation sont, à cet égard, particulièrement éloquents.

7 Bien qu'essentiels à la science dont ils constituent les moyens de production et de restitution, l'étude de l'instrumentation et de l'imagerie scientifique souffre d'un déficit d'outils méthodologiques en l'absence desquels leur inventaire et leur sauvegarde s'avèrent compromis. En effet, et alors que le patrimoine immobilier et une bonne part des éléments relevant du patrimoine mobilier peuvent, grâce aux Principes et Méthodes d'analyse du patrimoine culturel développés par l'Inventaire général, être étudiés et consultés sous forme de dossiers très détaillés et complets - de notices versées respectivement dans les bases documentaires Mérimée et Palissy - l'inventaire de l'instrumentation scientifique pose le plus souvent problème, malgré sa qualité de patrimoine mobilier. En répondant en effet à une terminologie à la fois très spécifique, abondante et croissante du fait de la diversité et de la technicité accrue des domaines scientifiques qu'elle concerne, l'instrumentation ne trouve pas aujourd'hui dans le Thésaurus de l'architecture, lexique de termes contrôlés d'indexation, toutes les occurrences nécessaires au renseignement des champs à mots-clés ${ }^{3}$ contenus dans les notices de la base Palissy. C'est pourquoi, et face à l'émergence de nouveaux domaines patrimoniaux dont celui de l'instrumentation scientifique dans la base Palissy, il semble primordial d'élargir la liste des termes autorisés pour l'indexation (descripteurs), voire de créer un thésaurus spécifique au patrimoine technique et scientifique afin d'en optimiser, sinon d'en créer, les moyens d'analyse, d'inventaire et de sauvegarde.

Indissociable, sémiologiquement, de l'instrumentation qu'elle met en quelque sorte en scène en permettant de comprendre ce pourquoi elle est faite et de visualiser les résultats qu'elle permet d'obtenir, l'imagerie scientifique pose, quant à son étude, un problème méthodologique assez complexe. N'ayant, en effet, aucune réalité matérielle au regard des Principes et Méthodes d'analyse du patrimoine culturel, l'imagerie scientifique qui peut être définie ici, par opposition à l'imagerie naturaliste, comme une synthèse démonstrative faite pour communiquer entre spécialistes, un mode de représentation codifié réunissant un ensemble de données exposées de manière didactique et pouvant également servir de vecteur à des concepts abstraits, ne peut donc être ni inventoriée, ni sauvegardée au titre du patrimoine historique. En raison de la place centrale qu'elle occupe dans l'histoire des sciences, il a semblé capital de lui rendre ce « corps » dont elle est aujourd'hui privée en substituant à sa fonction communicative purement référentielle 
une lecture centrée sur le signifié qu'elle provoque du point de vue des moyens techniques que nécessitent son acquisition et sa restitution.

La première phase de ce projet a consisté en l'élaboration d'un système descriptif de l'imagerie scientifique $e^{4}$ grâce auquel il a été possible d'identifier, de nommer et de classer les divers modes de représentation utilisés par les scientifiques pour exprimer et communiquer un résultat. Quatre niveaux de lecture successifs ont rythmé cette analyse.

10 Le niveau 1 de lecture, relatif aux caractéristiques physionomiques de l'imagerie publiée, a permis d'opérer une première distinction entre la figure dans le texte $e^{5}$, et la planche hors texte ${ }^{6}$, au regard de critères récurrents tels que l'emplacement de l'image dans une publication, ou le mode de formulation de sa légende.

\begin{tabular}{|l|l|}
\hline Matériel d'étude & Niveau 1 de lecture \\
\hline Imagerie scientifique & Figure dans le texte \\
\hline & Planche hors texte \\
\hline
\end{tabular}

11 Consacré à la reconnaissance des moyens techniques d'obtention d'un support visuel fixe, c'està-dire aux moyens ayant permis de restituer une image, indépendamment du contenu du message qu'elle véhicule, le niveau 2 de lecture a eu pour effet de classer les figures dans le texte et les planches hors texte en cinq types différents: l' image graphique ${ }^{7}$, l'image photographique ${ }^{8}$, l'image composée $e^{9}$, la planche graphique ${ }^{10}$, et la planche photographique ${ }^{11}$.

\begin{tabular}{|l|l|l|}
\hline Matériel d'étude & Niveau 1 de lecture & Niveau 2 de lecture \\
\hline & & Image graphique \\
\hline & Figure dans le texte & Image photographique \\
\hline Imagerie scientifique & & Image composée \\
\hline & Planche hors texte & Planche graphique \\
\hline & & Planche photographique \\
\hline
\end{tabular}

12 L'analyse des outils d'expression d'un contenu s'est efforcée, aux niveaux 3 et 4 de lecture, et à l'inverse du niveau 2, de saisir la corrélation existant entre la finalité des recherches menées par un scientifique et le mode d'expression visuelle qu'il va utiliser pour exprimer les résultats de ces recherches. Pour quelle raison, en effet, un scientifique va-til choisir d'illustrer ses travaux au moyen d'une image graphique plutôt que d'une image photographique ou d'une image composée? La réponse à cette interrogation se trouve dans la définition des huit outils d'expression d'un contenu identifiés au niveau 3 de lecture, et dont la formulation a permis de mettre en relief la vocation du $\operatorname{dessin}^{12}$, du graphique $^{13}$, de l'enregistrement graphique ${ }^{14}$, de la carte ${ }^{15}$ (images graphiques), ainsi que de la photographie ${ }^{16}$ (image photographique) dans leur fonction de restitution d'un contenu scientifique. Il apparait ainsi qu'à la spécificité de ces outils correspond un usage précis dicté par une finalité déterminée. La définition de l'image composée ${ }^{17}$, de la planche 
graphique $^{18}$ et de la planche photographique ${ }^{19}$ reprend les termes des différentes images graphiques et /ou photographiques (dessin, graphique, enregistrement graphique, carte, photographie) dont elles sont des combinaisons ou des juxtapositions.

\begin{tabular}{|l|l|l|l|}
\hline Matériel d'étude & $\begin{array}{l}\text { Niveau } \\
\text { lecture }\end{array}$ & de & Niveau 3 de lecture \\
\hline & & & Dessin \\
\hline $\begin{array}{l}\text { Imagerie } \\
\text { scientifique }\end{array}$ & Figure dans le texte & Image graphique & $\begin{array}{l}\text { Graphique } \\
\text { graphique }\end{array}$ \\
\hline & & Carte \\
\hline & Planche hors texte & Planche graphique & Planche graphique \\
\hline & & $\begin{array}{l}\text { Planche } \\
\text { photographique }\end{array}$ & Planche photographique \\
\hline
\end{tabular}

Certains des outils d'expression d'un contenu définis au niveau 3 de lecture ont dû être précisés par un niveau supplémentaire d'analyse, le niveau 4, du fait de la diversité des "formes" qu'ils revêtent et présentent à l'intérieur d'un même groupe typologique. Ainsi, le dessin a été subdivisé en dessin d'imitation ${ }^{20}$, en dessin semi-schématique ${ }^{21}$ et en schéma $^{22}$ et la carte en carte d'information ${ }^{23}$, en carte de localisation ${ }^{24}$, et en carte de résultats ${ }^{25}$. (fig. $n^{\circ} 4,5,6,7,8,9$ ) 
Figure 4

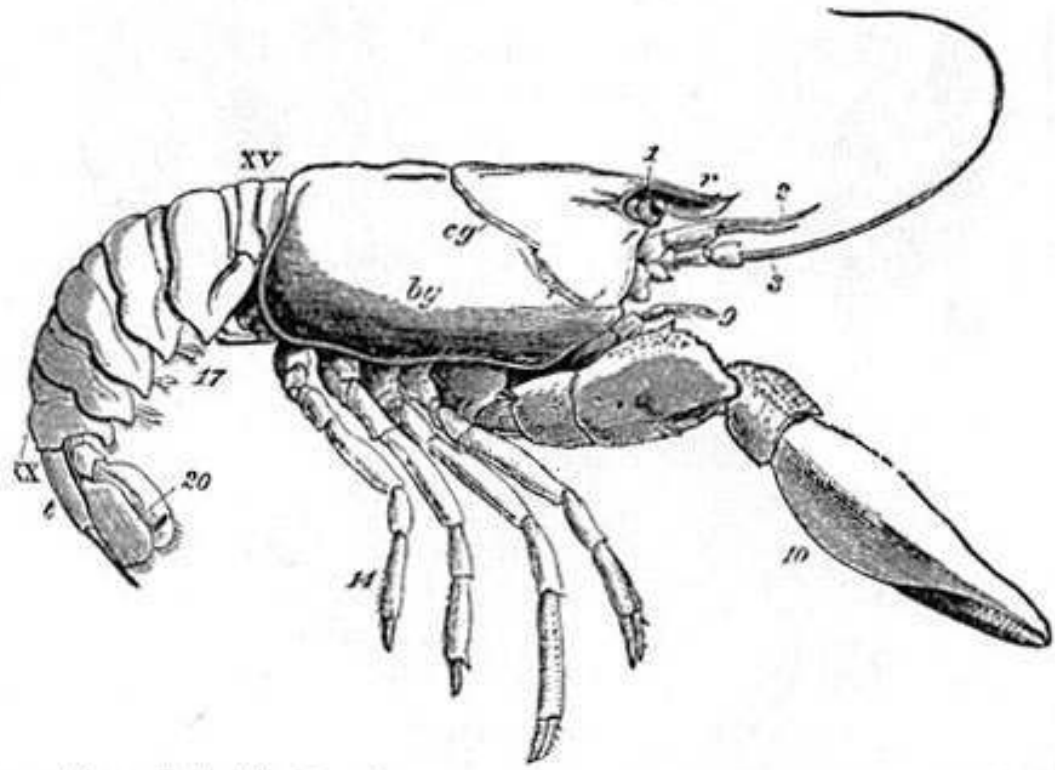

Fro. 2.- Astacus fluviatilis. Vun do proat d'un mâte (gr. nat.); bg, épimère ou branchiostégita Huxiey), séparcé antérieurement du tergum par le sillon cervical $\mathrm{cy} ; r$, rostre; $t$, telson; $t$, pó doncule de l'ceil; 2 , antennule; 3 , antenne; 9 , patte-máchoiro externe; 10 pince: 14 , dernitere patte-ambulatoire; 17 , fausse patte abdominale; 20 , lobe latéral de la nageoire eaudale; $\mathrm{XV}$, premier article de l'abdomon. (D'après M. Huxley.)

Dessin d'imitation. Crédit photographique : Vitzou A.-N., Recherches sur la structure et la formation des téguments chez les crustacés décapodes, in ARCHIVES DE ZOOLOgIE EXPÉRIMENTALE ET GÉNÉRALE, T. 10, 1882. Paris : Librairie de C. Reinwald, p. 540.

Figure 5

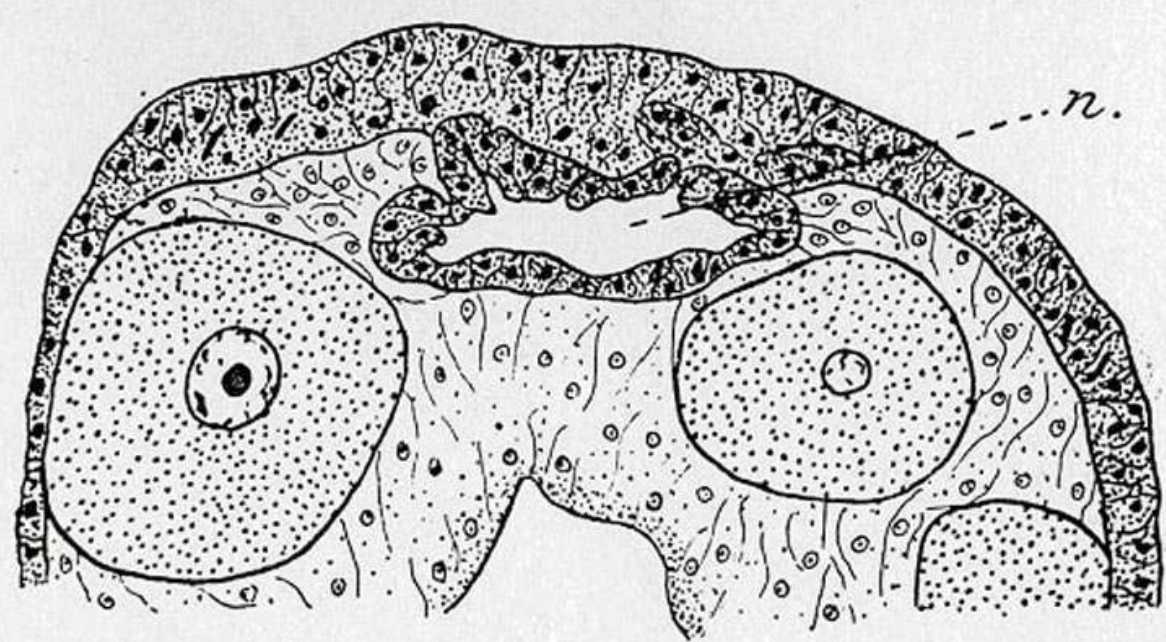

Fia. xxxix. Coupe d'un blastostyle femelle $(\times 240)$, le nodule médusaire $n$ est refermé.

Dessin semi-schématique. Crédit photographique : Teissier G., Recherches sur Dynamena Pumila (L.) fragments d'une monographie zoologique, in TRAVAUX DE LA STATION BIOLOgIQUE DE ROSCOFF, Fascicule 1, 30 avril 1923, Paris : Les Presses Universitaires de France, p. 42. 
Figure 6

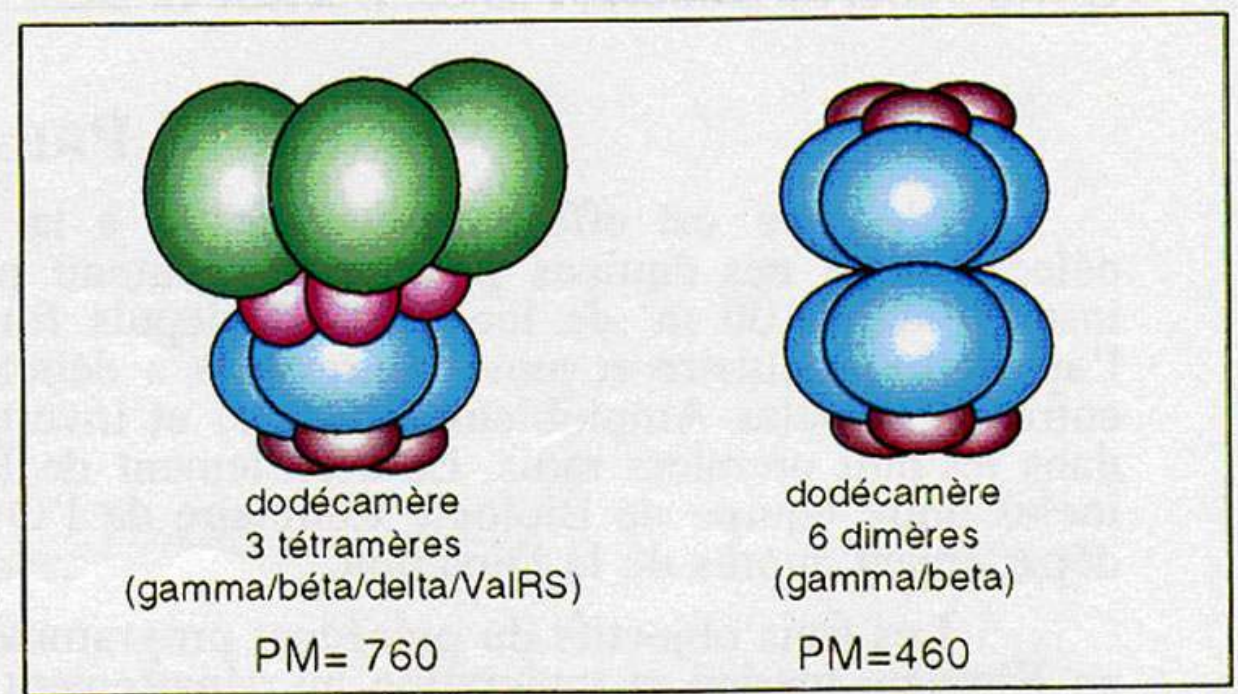

Modèle de structure quaternaire du complexe EF-1

Schéma. Crédit photographique : Bilan scientifique 1994-1998. Département de biologie cellulaire et moléculaire des modèles marins. Responsable Robert Bellé. Département 1. Équipe biologie cellulaire de l'ovocyte. Responsable Robert Bellé. Co-responsable Odile Mulner-Lorillon, in STATION BıoLogiquE DE ROSCOFF. COMITÉ SCIENTIFIQUE DES 28 ET 29 MAI 1998. DÉPARTEMENT DES SCIENCES DE LA VIE DU CNRS, UPR 9042. CENTRE d'ÉTUDES d'OCÉANOgRAPHIE ET DE BIOLOgIE MARINE. BILAN 1994-1998. PROJET 1999-2002. Mai 1998, p. 96.

Figure 7

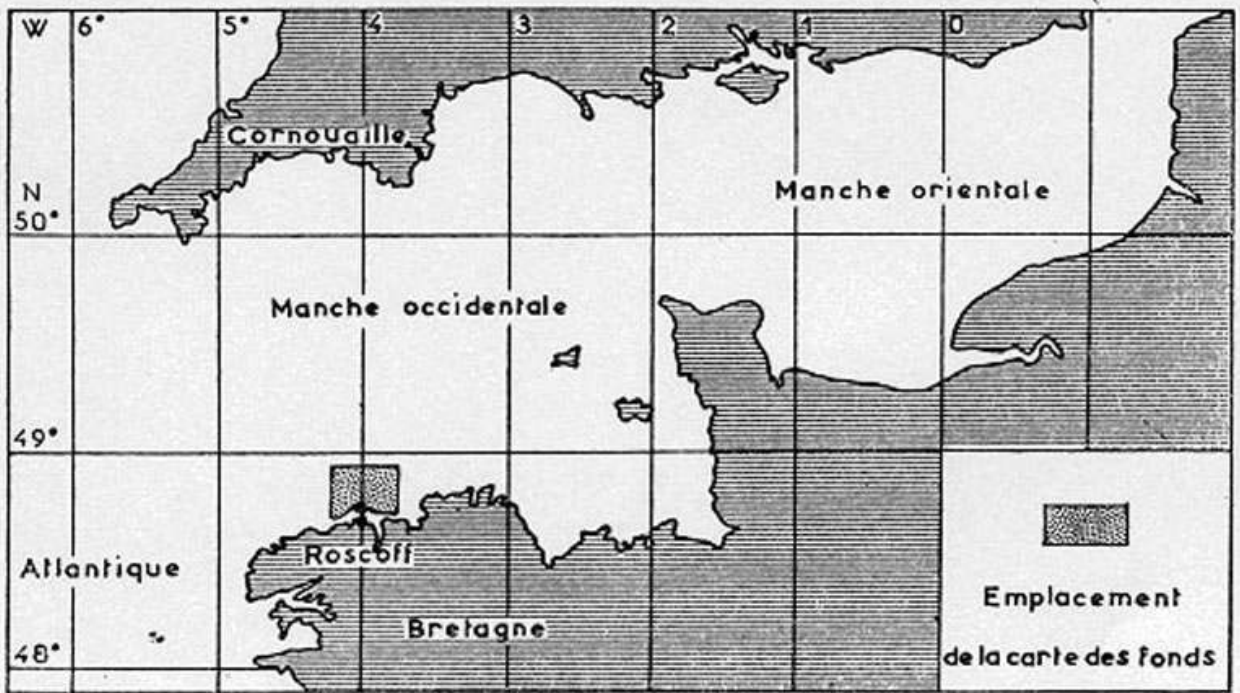

Figure 1

Situation géographique de Roscoff et emplacement de la carte des fonds.

Carte d'information. Crédit photographique : Boillot, G., La répartition des fonds sous-marins au large de Roscoff (Finistère), in CAHIERS De BIOLOgIE MARINE. Tome 1. Cahier 1. 1960. Éditions de la Station Biologique de Roscoff. Paris : Prieur et Robin imprimeurs, p. 4. 
Figure 8

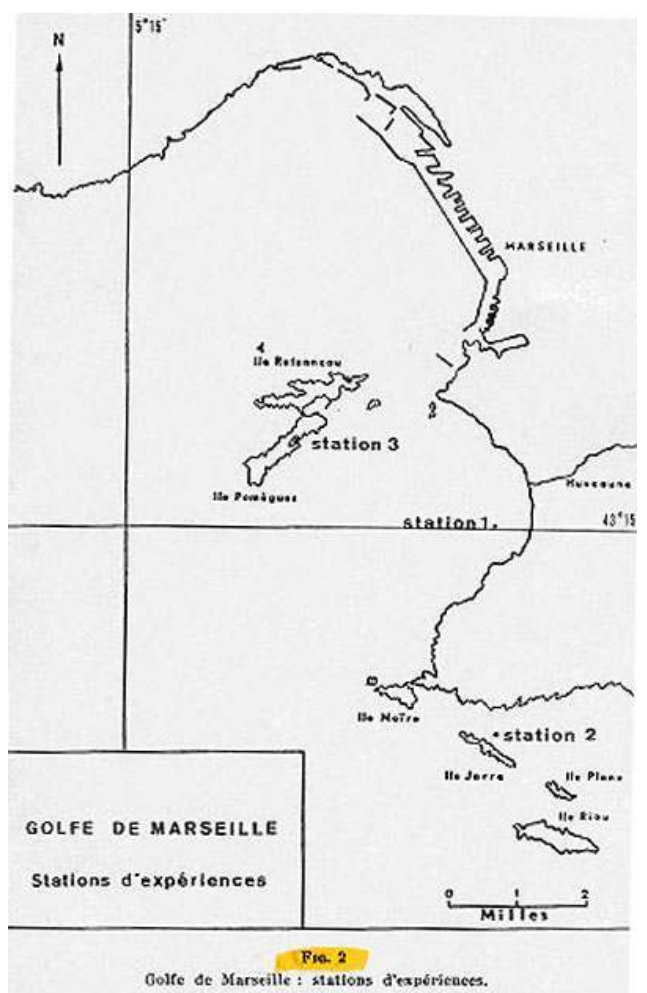

Carte de localisation. Crédit photographique : Guérin, J.P., Étude expérimentale de l'établissement d'un peuplement de substrat meuble à partir de larves méroplanctoniques, in CAHIERS DE BIOLOgIE MARINE , T. XI, Cahier 2, 1970. Éditions de la Station biologique de Roscoff. Paris : Robin \& Mareuge imprimeurs, p. 171. 
Figure 9
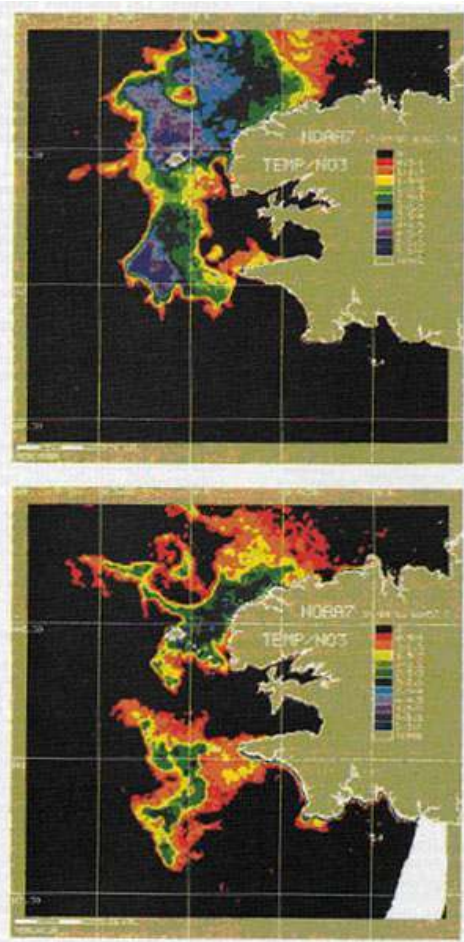

Carte de résultats. Crédit photographique : Bilan scientifique 1990-1994. Projet scientifique 1995-1998. Résultats scientifiques. Département océanographique et environnement côtier. Équipe chimie marine. Responsable Pascal Morin, in STATION BIOLOgIQUE DE ROSCOFF. COMITÉ SCIENTIfiQUE dU 2-3/06/1994 - BILAN 1990-1993, CENTRE d'ÉTUDES D'OCÉANOgRAPHIE ET DE BIOLOgIE MARINE, UPR 4601 DU DÉPARTEMENT DES SCIENCES DE LA VIE ET LABORATOIRE LACAZE-DUTHIERS, université Pierre et Marie Curie, p. 122.

14 Pour cette même raison de pluralité des modes d'expression d'un contenu, la photographie et l'image composée ont elles aussi été respectivement classées en plusieurs soustypologies : la photographie "commune ${ }^{26}$, la macrophotographie $e^{27}$ et la photomicrographi $e^{28}, l^{\prime}$ image composée graphique ${ }^{29}$ et l'image composée graphique et photographique $e^{30}$.(fig. $\mathbf{n}^{\circ} \mathbf{1 0}, \mathbf{1 1}$, $12,13,14)$ 
Figure 10

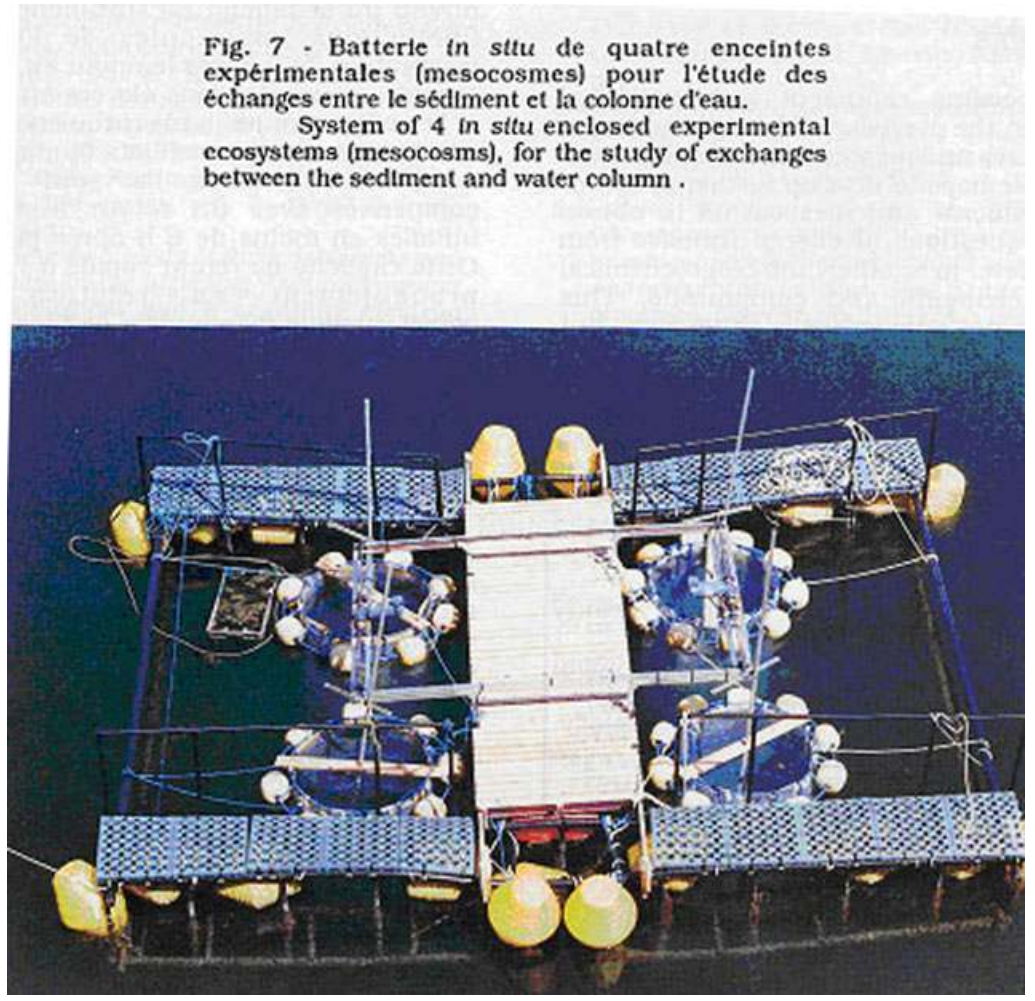

Photographie «commune ». Crédit photographique : Activités de recherche pour la période 1985-1990. Équipe écophysiologie et flux. Responsables P. Lasserre \& A. Toulmond. (1) Adaptations respiratoires des Annélides aquatiques. (2) Excrétion d'azote et adaptation à l'hypoxie de la macrofaune benthique. (3) Flux d'énergie microbiologique à l'interface eau- sédiment : approche micro-calorimétrique. (4) Effets de la bioturbation et d'une turbulence dans des écosystèmes expérimentaux, in OBSERVATOIRE OCÉANOLOgIQUE DE ROSCOFF. UNIVERSITÉ PIERRE ET MARIE CURIE-PARIS VI. CNRS. INSTITUT NATIONAL DES SCIENCES DE L'UNIVERS. RAPPORT DE LA STATION BIOLOgIQUE DE ROSCOFF 1985- 1990, p. 71. 
Figure 11

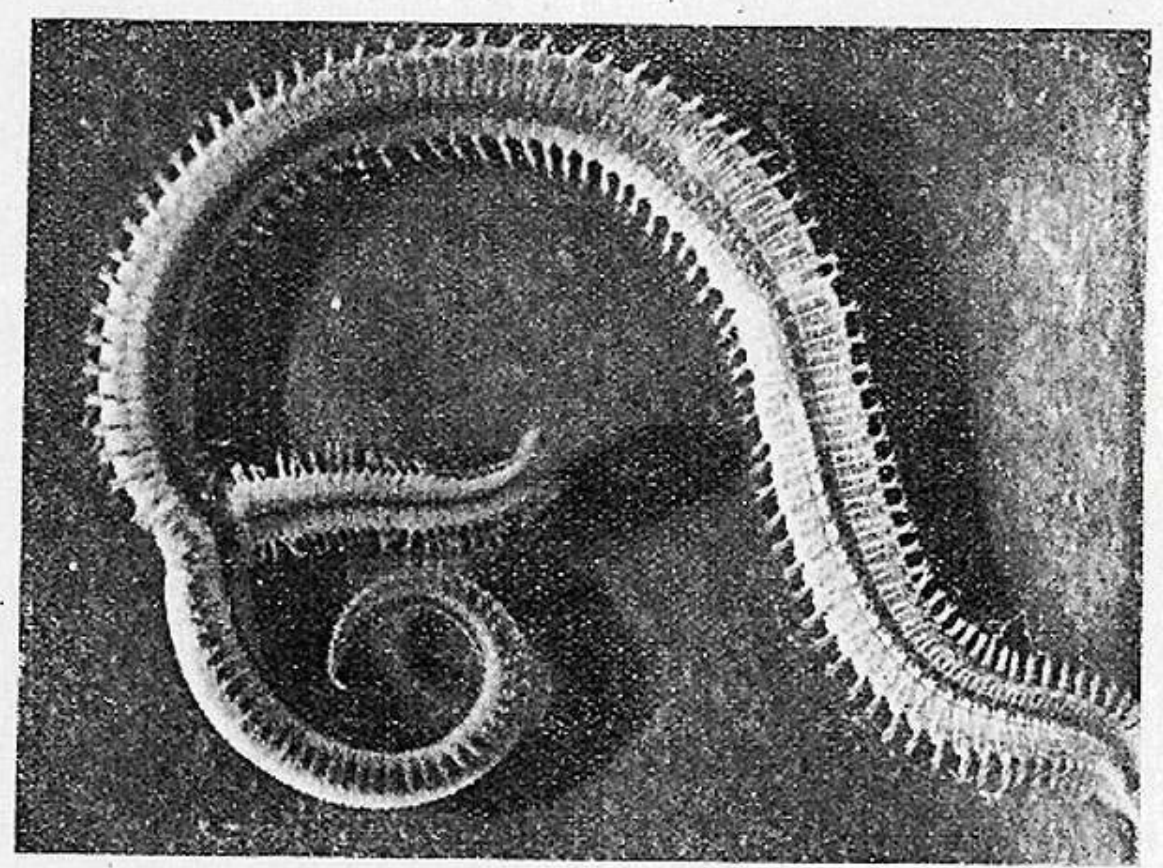

Frc. 1. - Photographie de la glycère $(\times 3)$.

Macrophotographie. Crédit photographique : RAPHAËL, C. Sur un cas de bifurcation postérieure chez Glycera Convoluta, in travaUX de LA STATION BIologique De Roscoff, Fascicule 11, $7^{\text {er }}$ décembre 1933. Paris : Les Presses Universitaires de France, p. 22. 
Figure 12

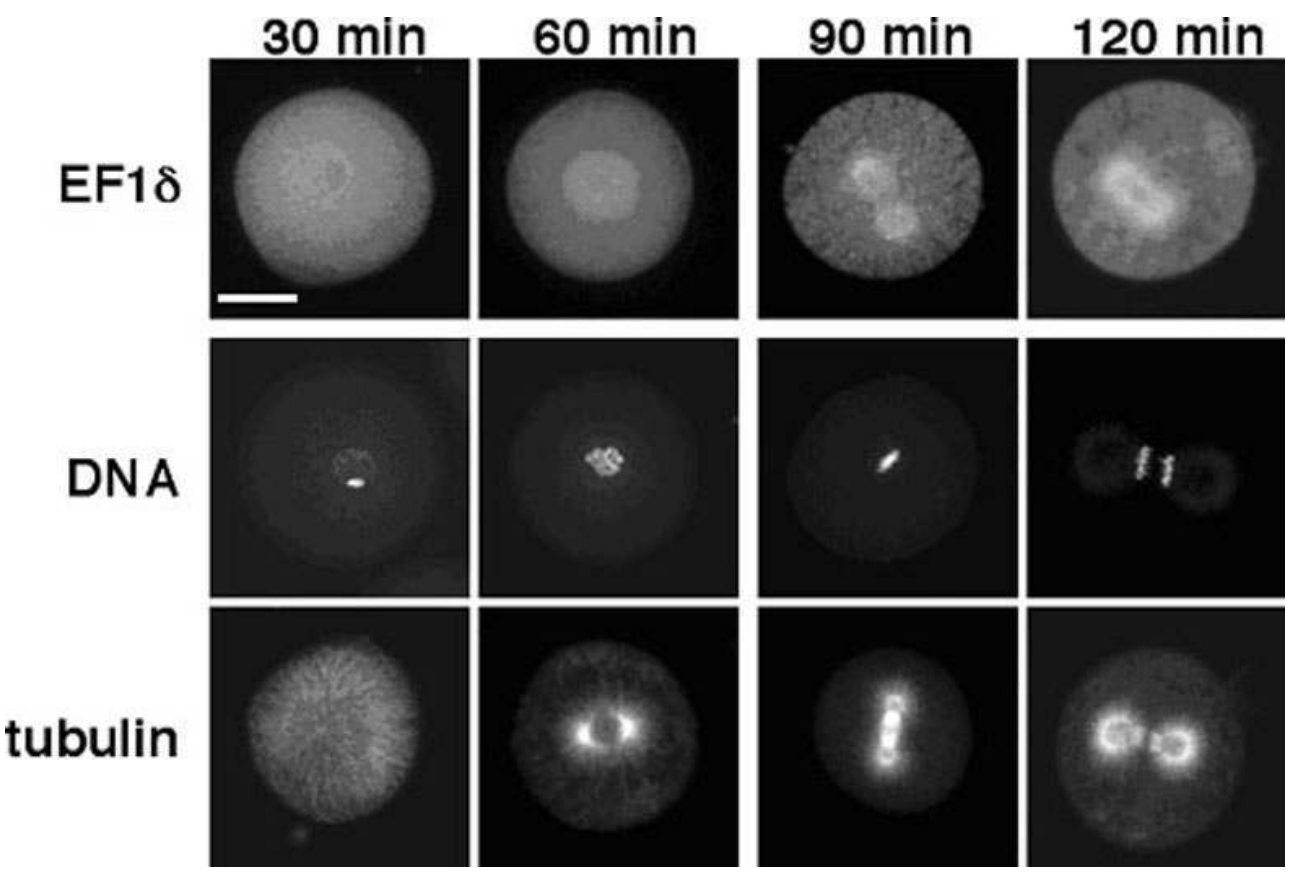

Photomicrographie. Crédit photographique : Bilan scientifique pour la période 1999-2003. Septembre 2003. Équipe «Cycle Cellulaire et Développement ». Responsables Robert Bellé \& Odile MulnerLorillon, in UNIVERSITÉ PIERRE ET MARIE CURIE-PARIS VI. DEMANDE DE CRÉATION. UMR CNRS-UPMC. " MER \& SANTÉ ", sans $n^{\circ}$ de page. 
Figure 13

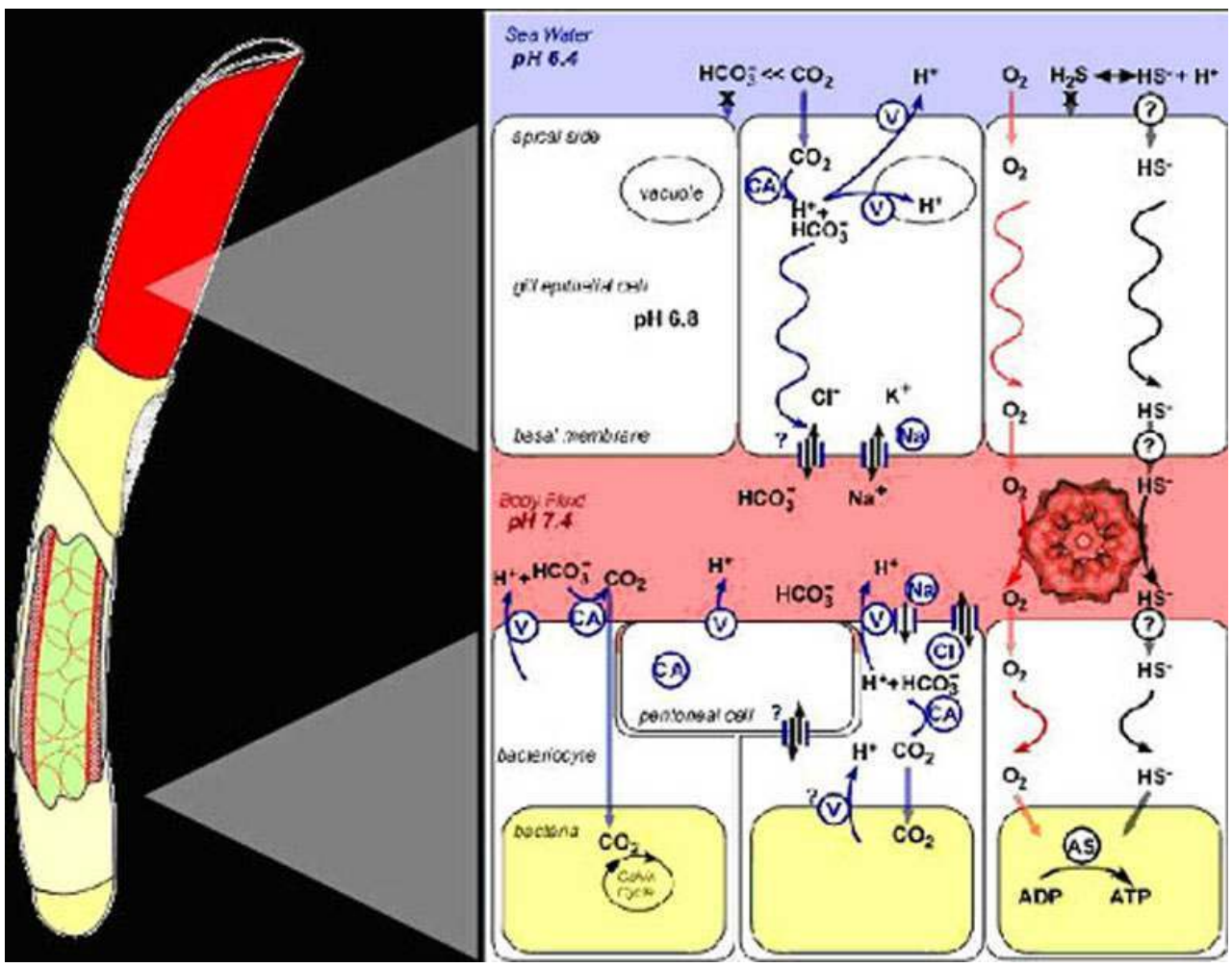

Image composée graphique. Crédit photographique : Equipe Ecophysiologie. Responsable François Lallier. Thématiques de recherche développées par l'équipe. Rapport scientifique concis 2000-2003, in UNIVERSITÉ PIERRE ET MARIE CURIE-PARIS VI. DEMANDE DE CRÉATION. UMR CNRS-UPMC. " ADAPTATION ET DIVERSITÉ EN MILIEU MARIN ». AD2M. CNRS SDV. SECTIONS CN 30/12. OCTOBRE 2003, p. 78 . 
Figure 14

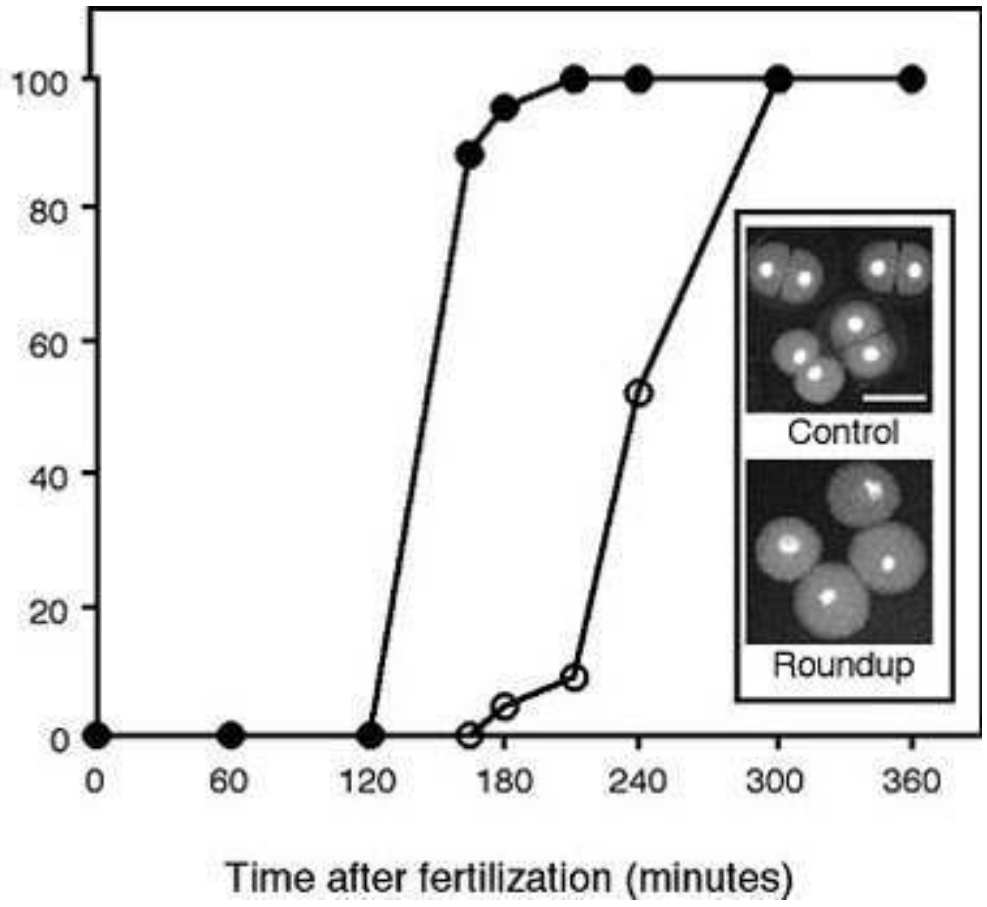

Image composée graphique et photographique. Crédit photographique : Équipe «Cycle Cellulaire et Développement ». Responsables Robert Bellé \& Odile Mulner-Lorillon, in UNIVERSITÉ PIERRE ET MARIE CURIE-PARIS VI. DEMANDE DE CRÉATION.UMR CNRS-UPMC. “ MER \& SANTÉ ». BILAN SCIENTIfIQUE POUR LA PÉriode 1999-2003. SEPTEMBre 2003, sans $n^{\circ}$ de page.

15 L'analyse des images entrant dans les typologies du graphique, de l'enregistrement graphique, de la planche graphique et de la planche photographique, n'ont motivé, ainsi que l'indique le tableau récapitulatif ci-après, aucun niveau d'analyse supplémentaire. (fig. $\mathbf{n}$ - $15,16,17,18)$ 


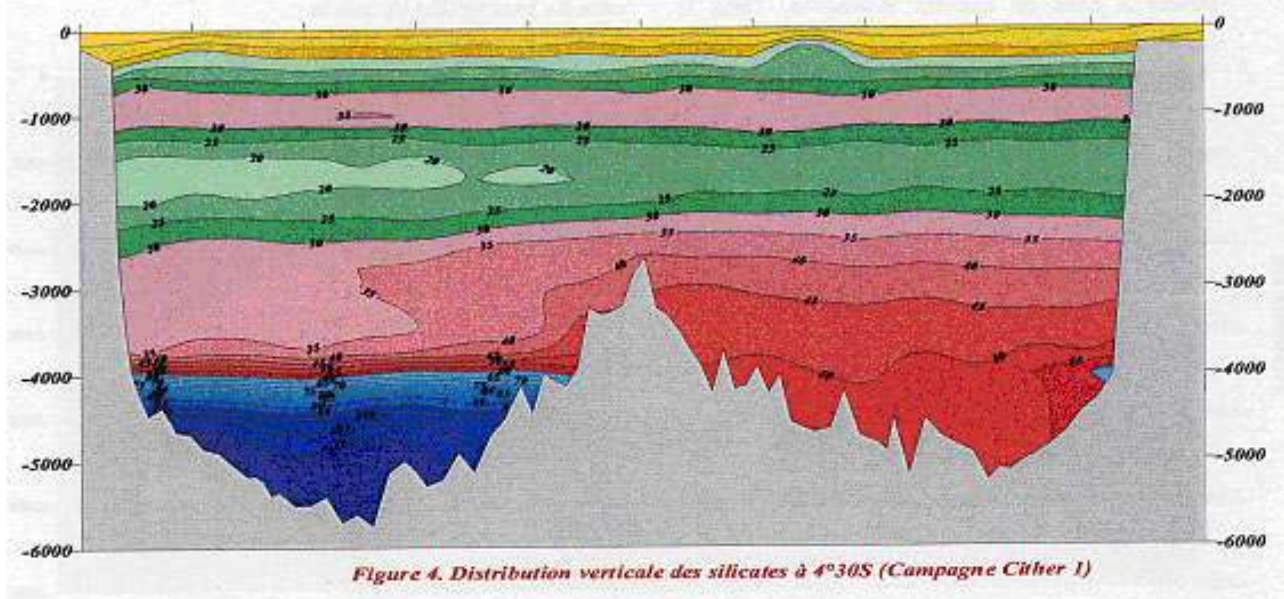

Graphique. Crédit photographique : Bilan scientifique 1994-1998. Département océanographie et environnement côtier. Responsable Franck Gentil. Département 3. Équipe chimie marine. Responsables Pascal Morin et Pierre Le Corre. Observatoire océanologique de Roscoff. Comité scientifique des 28 et 29 mai 1998, in DÉPARTEMENT DES SCIENCES DE LA VIE DU CNRS, UPR 9042. CENTRE D'ÉTUDES D'OCÉANOgRAPHIE ET DE BIOLOgIE MARINE. BILAN 1994-1998. PROJET 1999-2002. MAI 1998, p. 179.

Figure 16

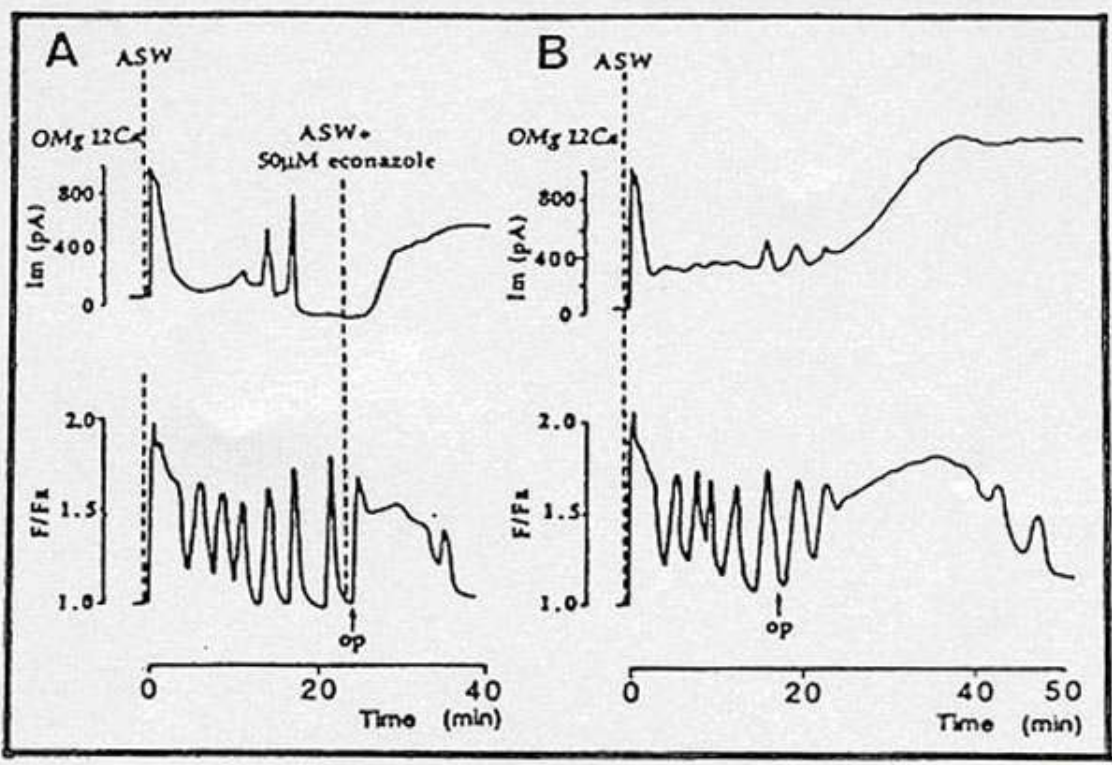

Fig. 4 : Enregistrements simultanés du signal de fluorescence et du courant transmembranaire d' ovocytes de crevette activés par $\mathrm{Mg}^{2+}$ de l'eau de mer (ASW). En (A) Effets de l'éconazole (50 $\mu \mathrm{M}$ dans ASW); (B) Enregistrements témoins en ASW.

Enregistrement graphique. Crédit photographique : Bilan scientifique 1994-1998. Département de biologie cellulaire et moléculaire des modèles marins. Responsable Robert Bellé. Département 1. Équipe canaux ioniques et développement d'animaux marins. Responsable Henri Goudeau. Station biologique de Roscoff. Comité scientifique des 28 et 29 mai 1998, in DÉPARTEMENT DES SCIENCES DE LA VIE DU CNRS, UPR 9042. CENTRE D'ÉTUDES D'OCÉANOgRAPHIE ET DE BIOLOgIE MARINE. BILAN 1994-1998. PROJET 1999-2002. MAI 1998, p. 89. 
Figure 17

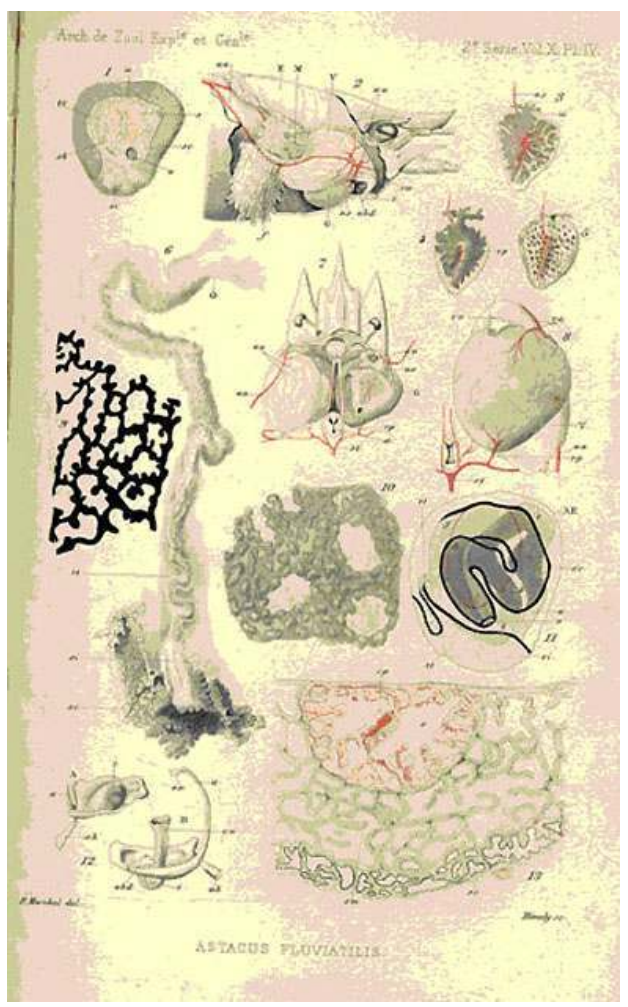

Planche graphique. Crédit photographique : MARCHAL, P. Recherches anatomiques et physiologiques sur l'appareil excréteur des crustacés décapodes, in ARCHIVES DE ZOOLOgIE EXPÉRIMENTALE ET GÉnérale, 2e série, T. X, 1892. Paris : Librairie de C. Reinwald, PI IV. 
Figure 18

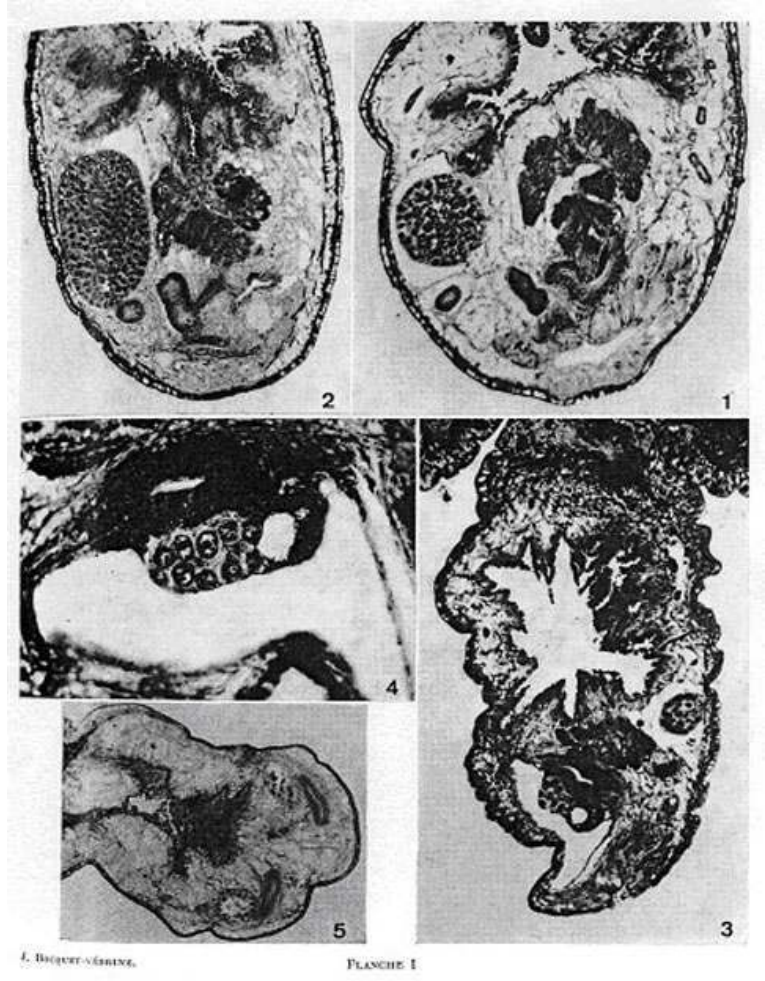

Planche photographique. Crédit photographique : BOCQUET, C., BOCQUET-VÉDRINE, J. et L'HARDY, J.P. Contribution à l'étude du développement des organes génitaux chez Xenocoelama alleni (Brumpt), Copépode parasite de Polycirrus caliendrum Claparède, in CAHIERS DE BIOLogIE MARINE, T. XI, Cahier 2, 1970. Editions de la Station Biologique de Roscoff. Paris : Robin \& Mareuge imprimeurs, PI I.

\begin{tabular}{|l|l|l|l|l|}
\hline $\begin{array}{l}\text { Matériel } \\
\text { d'étude }\end{array}$ & $\begin{array}{l}\text { Niveau 1 de } \\
\text { lecture }\end{array}$ & $\begin{array}{l}\text { Niveau 2 de } \\
\text { lecture }\end{array}$ & $\begin{array}{l}\text { Niveau 3 de } \\
\text { lecture }\end{array}$ & Niveau 4 de lecture \\
\hline & & & & d'imitation \\
\hline & & & Dessin & Semi-schématique \\
\hline & & Image graphique & $\begin{array}{l}\text { Enregistrement } \\
\text { graphique }\end{array}$ & Schéma \\
\hline & & & & Graphique \\
\hline $\begin{array}{l}\text { Imagerie } \\
\text { scientifique }\end{array}$ & $\begin{array}{l}\text { Figure dans } \\
\text { le texte }\end{array}$ & & Carte & de localisation \\
\hline & & & & de résultats \\
\hline
\end{tabular}




\begin{tabular}{|l|l|l|l|l|}
\hline & & $\begin{array}{l}\text { Image } \\
\text { photographique }\end{array}$ & Photographie & Macrophotographie \\
\hline & & & & Photomicrographie \\
\hline & & Image composée & Image composée & Graphique \\
\hline & $\begin{array}{l}\text { Planche } \\
\text { hors texte }\end{array}$ & Planche graphique & Planche graphique & $\begin{array}{l}\text { Graphique } \\
\text { photographique }\end{array}$ \\
\hline & & $\begin{array}{l}\text { Planche } \\
\text { photographique }\end{array}$ & $\begin{array}{l}\text { Planche } \\
\text { photographique }\end{array}$ & \\
\hline
\end{tabular}

Réalisée en étroite collaboration avec les chercheurs de la Station biologique de Roscoff, la première phase du projet de «re-matérialisation» de l'imagerie scientifique a vu la mise en place d'une nomenclature et d'un classement typologique du corpus qui, s'ils ont apporté un contenu autre que purement documentaire à l'image scientifique, ont également servi de fondements à la seconde phase du projet : l'élaboration d'une méthode d'inventaire de l'illustration scientifique. Inspirée à la fois du Système descriptif de l'Illustration ${ }^{31}$, de systèmes descriptifs bibliographiques ${ }^{32}$ et de notices contenues dans la base $\operatorname{Archidoc}^{33}$, cette méthode dont l'unité documentaire (le support de référencement) est l'illustration scientifique ${ }^{34}$ repose sur quatre domaines d'analyse.

Le premier domaine d'analyse, intitulé désignation du support de l'illustration scientifique, ne contient qu'un seul champ consacré à la dénomination de l'objet. S'il permet de préciser la nature du document écrit dont est extraite l'illustration scientifique, son renseignement a entraîné la création d'un premier lexique hiérarchisé.

\section{Lexique dénomination de l'objet}

(Exemples de descripteurs)

$-<$ image scientifique $>=<$ illustration scientifique $>$

$--<$ document écrit publié $>$

--- article

--- actes de colloque

--- livre

--- rapport scientifique

--- thèse $=<$ mémoire universitaire $>$

--- tiré à part

Le deuxième domaine d'analyse, références du support de l'illustration scientifique, contient cinq champs permettant d'indiquer les références bibliographiques du document dont est extraite l'illustration, l'immatriculation du document écrit et les droits de diffusion, le lieu de conservation du document, et sa cote de conservation.

Le troisième domaine, consacré à la description de l'illustration scientifique, contient douze champs grâce auxquels la nature de l'illustration, son auteur, l'auteur de la reproduction, le format de la reproduction, la localisation de l'illustration, son numéro d'ordre, sa légende, sa taille initiale, sa couleur, sa dénomination, sa classification, et son intérêt vont être précisés. Trois 
des douze champs - les champs auteur, dénomination et classification - ont également nécessité la création de lexiques hiérarchisés. Les descripteurs du premier lexique auteur de l'illustration - vont servir, à l'image des descripteurs contenus dans le lexique AUTR grâce auxquels la profession du créateur d'une œuvre ou du constructeur d'une machine peuvent être mentionnés dans la base Palissy, à indiquer la qualité (ou la profession) de l'auteur de l'illustration scientifique.

\section{Lexique auteur de l'illustration}

(Exemples de descripteurs)

- - scientifique

- - - biologiste

- - - océanographe

- - - zoologiste

Le lexique commun aux champs dénomination de l'objet et classification de l'objet consiste en une synthèse des recherches menées dans le cadre du système descriptif de l'imagerie scientifique. Les descripteurs qui y sont consignés reprennent en effet la hiérarchie et la nomenclature alors définies pour identifier et classer l'imagerie scientifique.

\section{Lexique dénomination et classification de l'illustration}

(Descripteurs)

$-<$ illustration scientifique $>$

- - <igure dans le texte>

- - - <image graphique>

- - - - dessin

- . - - - dessin d'imitation

- - - - dessin semi-schématique

- - - - schéma

- - - graphique

- - - enregistrement graphique

- - - - carte

- - - - carte d'information

- - - - carte de localisation

- - - - - carte de résultats

- - - <image photographique>

- - - - photographie

- - - - photographie « commune»

- - - - macrophotographie

- - - - microphotographie

- - - <image composée>

- - - image composée graphique

- - - - image composée graphique et photographique

- - <planche hors texte>

- - - <planche graphique>

- - - <planche photographique>

24 Le quatrième et dernier domaine d'analyse, liens, a pour vocation de préciser le contexte historique, technique, culturel, etc., dans lequel l'illustration scientifique a été produite, en conduisant, par un système de liens informatiques, au patrimoine architectural et /ou mobilier contenu dans les bases documentaires Mérimée et /ou Palissy. Ce domaine est 
extrêmement important dans la mesure où il fait le lien entre les trois types d'éléments constitutifs du patrimoine scientifique : le patrimoine immobilier, le patrimoine mobilier et l'imagerie scientifique que l'on peut à présent qualifier de patrimoine iconographique scientifique.

\begin{tabular}{|c|c|}
\hline $\begin{array}{l}\text { Méthode d'analyse et d'inventaire de } \\
\text { l'illustration scientifique }\end{array}$ & (Présentation succincte) \\
\hline $\begin{array}{l}\text { Domaine 1: Désignation du support de } \\
\text { l'illustration scientifique }\end{array}$ & \\
\hline $\begin{array}{l}1 \text { champ } \\
\text { dénomination de l'objet }\end{array}$ & $\begin{array}{l}\text { Création d'un lexique hiérarchisé : } \\
\text { dénomination de l'objet }\end{array}$ \\
\hline $\begin{array}{l}\text { Domaine 2: Références du support de } \\
\text { l'illustration scientifique }\end{array}$ & \\
\hline $\begin{array}{l}5 \text { champs } \\
\text { références bibliographiques } \\
\text { immatriculation du document écrit } \\
\text { droits de diffusion } \\
\text { lieu de conservation du document } \\
\text { cote de conservation }\end{array}$ & \\
\hline $\begin{array}{l}\text { Domaine 3: Description de l'illustration } \\
\text { scientifique }\end{array}$ & \\
\hline $\begin{array}{l}12 \text { champs } \\
\text { nature de l'illustration } \\
\text { auteur } \\
\text { auteur de la reproduction } \\
\text { format de la reproduction } \\
\text { localisation de l'illustration } \\
\text { numéro d'ordre } \\
\text { légende } \\
\text { taille initiale } \\
\text { couleur } \\
\text { dénomination } \\
\text { classification } \\
\text { intérêt }\end{array}$ & $\begin{array}{l}\text { Création de deux lexiques hiérarchisés: } \\
\text { auteur de l'illustration } \\
\text { dénomination de l'objet } \\
\text { classification de l'objet }\end{array}$ \\
\hline Domaine 4 : Liens & \\
\hline $\begin{array}{l}2 \text { champs } \\
\text { Base Mérimée } \\
\text { Base Palissy }\end{array}$ & \\
\hline
\end{tabular}


Appliquées aux domaines de la biologie marine et de l'océanographie, les recherches sur l'imagerie scientifique n'ont, à ce stade de la réflexion, aucune prétention d'exhaustivité quant aux conclusions qui en émanent. Le système descriptif de l'imagerie scientifique ainsi que sa méthode d'analyse et d'inventaire sont en effet avant tout des prototypes d'outils qui demandent désormais à être expérimentés, perfectionnés et étendus à d'autres domaines de la science, mais grâce auxquels une dynamique a été créée. Si le propos était certes d'entreprendre une démarche visant à rendre un « corps » à l'image scientifique afin de pouvoir l'analyser et la sauvegarder en tant que témoin historique et patrimonial majeur de l'histoire des sciences, d'autres enjeux tout aussi importants justifiaient les recherches.

Face à l'extraordinaire engouement du public pour la science ainsi qu'en témoignent, depuis quelques années, les diverses initiatives de diffusion de la culture scientifique telles que cycles de conférences, expositions, visites d'établissements de recherche, ateliers pédagogiques, etc., il paraît en effet primordial de constituer, en amont de cette politique d'animation, un réservoir de connaissances, base et « matière première » d'une communication pertinente et efficiente. C'est la raison pour laquelle la création d'outils méthodologiques propres à analyser, inventorier et sauvegarder le patrimoine scientifique "dans tous ses états", c'est-à-dire au regard de la spécificité et de la pluridisciplinarité qui le caractérise, s'avère être une démarche primordiale d'un point de vue non seulement historique mais aussi, et peut-être surtout, d'un point de vue didactique.

\section{NOTES}

1. Site de la Station Biologique de Roscoff : http://www.sb-roscoff.fr

2. GUESNIER, Valérie. Les images de la Station biologique de Roscoff des années 1870 à nos jours. Patrimoine et images d'un patrimoine à sauvegarder pour communiquer, Thèse de doctorat en histoire et civilisations, option histoire des sciences, sous la direction de Jean Dhombres. Paris : EHESS, mars 2007.

3. La désignation et la description de l'instrumentation scientifique sont directement concernées.

4. Ce "système descriptif de l'imagerie scientifique " a été élaboré à partir de l'analyse d'un corpus de près de 1300 images puisées dans les publications de la Station biologique de Roscoff : Les Archives de Zoologie Expérimentale et Générale (années 1872, 1882, 1892, 1902, 1911-12) ; Les Travaux de la Station Biologique de Roscoff (années 1923, 1933); Les Travaux de la Station Biologique de Roscoff, Nouvelle Série (année1950) ; Les Cahiers de Biologie Marine (années 1960, 1970) ; ainsi que dans ses Bilans quadriennaux d'établissement (années 1985-1990, 1990-1994, 1994-1998, 1999-2003).

5. La figure dans le texte est une image insérée dans un texte, accompagnée d'une légende dont les premiers termes lui attribuent l'abréviation Fig. suivie d'une numérotation. Une même figure peut parfois comprendre plusieurs images qui reçoivent alors une sous-numérotation.

6. La planche hors texte consiste en un ensemble d'images regroupées sur une ou deux pages généralement placées en vis-à-vis et accompagnées d'une légende dont les premiers termes lui attribuent l'abréviation $P l$. suivie d'une numérotation. La légende est le plus souvent séparée de 
la planche, et les planches habituellement placées en fin d'article ou regroupées en fin de publication.

7. L'image graphique est une image analogique ou numérique obtenue par la technique du dessin réalisé soit manuellement, soit au moyen d'appareils enregistreurs, soit par le biais d'un logiciel graphique.

8. L'image photographique est une image analogique ou numérique obtenue par les procédés argentiques ou numériques de la photographie.

9. L'image composée est une image analogique ou numérique résultant de l'assemblage de deux images graphiques, ou de la combinaison d'une image graphique à une image photographique.

10. La planche graphique est un ensemble d'images analogiques ou numériques obtenues par la technique du dessin réalisé soit manuellement, soit au moyen d'appareils enregistreurs, soit par le biais d'un logiciel graphique, regroupées sur une ou deux pages généralement placées en vis-àvis.

11. La planche photographique est un ensemble de représentations de taille généralement plus petite ou plus grande que nature d'un modèle, obtenues par les procédés argentiques ou numériques de la photographie, regroupées sur une ou deux pages généralement placées en visà-vis, accompagnée d'une légende le plus souvent séparée de la planche.

12. Le dessin est l'expression figurée de modèles réels ou de données abstraites au moyen de formes pouvant aller de la plus simple à la plus complexe.

13. Le graphique est la traduction imagée de données chiffrées au moyen de lignes, de figures stylisées.

14. L'enregistrement graphique est une opération purement mécanique, sans travail de calcul préalable, consistant en une transcription de mesures physiques, par un appareil, sur un support matériel.

15. La carte est la représentation à échelle réduite de la surface totale ou partielle du globe terrestre.

16. La photographie est la représentation généralement plus petite ou plus grande que nature d'un modèle, obtenue à partir des procédés argentiques ou numériques de la photographie.

17. L'image composée est l'expression d'un résultat au moyen d'une image assemblant entre elles deux images graphiques de même type - dessin, graphique, enregistrement graphique, carte - ou de types différents; ou combinant l'une de ces images graphiques à une photographie.

18. La planche graphique est un ensemble d'images analogiques ou numériques telles que dessins, graphiques, enregistrements graphiques ou cartes, regroupées sur une ou deux pages généralement placées en vis-à-vis, accompagnées d'une légende le plus souvent séparée de la planche.

19. La planche photographique est un ensemble de représentations de taille généralement plus petite ou plus grande que nature d'un modèle, obtenues par les procédés argentiques ou numériques de la photographie, regroupées sur une ou deux pages généralement placées en visà-vis, accompagnée d'une légende le plus souvent séparée de la planche.

20. Le dessin d'imitation relève d'une démarche descriptive. Il est la transcription exacte de l'ensemble des caractères constitutifs et observables, soit à l'œil nu, soit à l'aide d'un appareil optique, d'un modèle généralement pris hors de son contexte écologique.

21. Le dessin semi-schématique relève d'une démarche descriptive. Il est la représentation partielle des caractères constitutifs et observables, soit à l'œil nu, soit à l'aide d'un appareil optique, d'un modèle généralement pris hors de son contexte écologique. C'est un dessin dépouillé de ce qui n'est pas essentiel afin de mettre en relief les caractères sur lesquels il convient d'insister.

22. Le schéma relève d'une démarche interprétative. Il est la restitution, réduite à quelques formes simplifiées, des résultats d'une investigation portant soit sur des modèles réels préalablement observés, soit sur des données abstraites telles que concepts, théories, phénomènes, processus. 
23. La carte d'information est un document préexistant permettant de situer une zone géographique.

24. La carte de localisation permet, par les informations qui y sont consignées, de désigner certains lieux géographiques précis.

25. La carte de résultats exprime la synthèse de phénomènes observés, ou de résultats obtenus, dans une zone géographique déterminée.

26. La photographie "commune» est la représentation généralement plus petite que nature du modèle qu'elle représente.

27. La macrophotographie est la photographie très rapprochée de petits modèles donnant une image plus grande que nature.

28. La photomicrographie est la photographie d'un modèle observé au microscope optique ou électronique.

29. L'image composée graphique est une image assemblant entre elles deux images graphiques relevant de l'un ou l'autre des différents types définis aux niveaux 3 et 4 de lecture.

30. L'image composée graphique et photographique est une image combinant une image graphique relevant de l'un ou l'autre des différents types définis aux niveaux 3 et 4 de lecture, à une photographie " commune », une macrophotographie ou une photomicrographie.

31. DESSAUX, Christophe (dir.). Système descriptif de l'illustration, Documents \& Méthodes hors-série. Paris : Inventaire Général, Editions du patrimoine, 1999.

32. Logiciel de bibliothèque Biblio-tech, et base de données Current contents.

33. Base de données Archidoc (puis onglet Bibliographie), ministère de la Culture et de la Communication - direction de l'Architecture et du Patrimoine.

34. Par illustration scientifique l'on entend toute image venant en illustration d'un texte scientifique écrit et publié. Ce choix a été imposé par la nature du corpus étudié.

\section{RÉSUMÉS}

Mise en relief par des travaux de recherche sur les images de la Station biologique de Roscoff, des années 1870 à nos jours, l'importance de l'imagerie scientifique au sein du patrimoine scientifique, a conduit à élaborer un système de lecture descriptif de ce corpus iconographique spécifique qui, en l'absence d'une méthode d'analyse, ne peut être aujourd'hui inventorié au titre $\mathrm{du}$ patrimoine historique et culturel national.

This article is inspired by some research carried out on the images held by the Roscoff biological station from 1870 up to the present day. The importance of this scientific imagery, within the broader context of the scientific heritage as a whole, led to the establishment of a new descriptive system for the corpus. Without such an analytical tool, it is difficult to draw up inventories of such images and to integrate them into the national cultural heritage. 


\section{INDEX}

Mots-clés : station biologique de Roscoff, imagerie scientifique, image scientifique, illustration scientifique, dessin d'imitation, dessin semi-schématique, schéma, carte d'information, carte de localisation, carte de résultats, macrophotographie, photomicrographie, image composée graphique, image composée graphique et photographique, planche photographique, planche graphique

Keywords : roscoff biological station, scientific imagery, scientific image, scientific illustration, imitative drawing, half-schematic drawing, diagram, information map, location map, macrophotography, photomicrography, composed graphic image, composed graphic and photographic image, photographic sheet, graphic sheet

\section{AUTEUR}

\section{VALÉRIE GUESNIER}

Docteur en histoire et civilisations, chargée de mission à l'action culturelle, Pays touristique du Léon, Saint-Pol de Léon. valerie.guesnier@aliceadsl.fr 